\title{
Race/Ethnicity and Patient Satisfaction
}

\section{Using the Appropriate Method to Test for Perceived Differences in Care}

Donald A. Barr, MD, PhD

OBJECTIVE: To determine whether an established patient satisfaction scale commonly used in the primary care setting is sufficiently sensitive to identify racial/ethnic differences in satisfaction that may exist; to compare a composite indicator of overall patient satisfaction with a 4-item satisfaction scale that measures only the quality of the direct physician-patient interaction.

DESIGN: Real-time survey of patients during a primary care office visit.

SETTING: Private medical offices in a generally affluent area of northern California.

PARTICIPANTS: Five hundred thirty-seven primary care patients selected at random from those entering a medical office.

MAIN OUTCOME MEASURES: Patient satisfaction using 1) a composite, 9-item satisfaction scale (VSQ-9); and 2) a 4-item subset of that scale that measures only satisfaction with direct physician care.

RESULTS: The 9-item, composite scale identified no significant difference in patient satisfaction between white and nonwhite patients, after controlling for patient demographics and other aspects of the visit. The 4-item, physician-specific scale indicated that nonwhite patients were less satisfied than white patients with their direct interaction with the physicians included in the study $(P \leq .01)$.

CONCLUSIONS: Measurements of patient satisfaction that use multi-item, composite indicators should also include focused comparisons of satisfaction directly with the care provided by the physician. In measurements of patient satisfaction, patient race/ethnicity should be included as an explanatory variable. The results also confirm earlier findings that factors external to the direct physician-patient interaction can have substantial effects on patients' perceptions of that interaction.

KEY WORDS: health disparities; quality of care; primary care; race; ethnicity.

J GEN INTERN MED 2004;19:937-943.

I n its 2002 report Unequal Treatment, the Institute of Medicine concluded that "racial and ethnic minority patients are found to receive a lower quality and intensity

Received from the Department of Sociology (DAB), Stanford University, Stanford, Calif.

These results were presented to a session of the Section on Medical Care of the American Public Health Association as part of the American Public Health Association annual meeting in November 2002.

Address correspondence and requests for reprints to Dr. Barr: Building 80, MC 2160, Stanford University, Stanford, CA 94305-2160 (e-mail: barr@stanford.edu). of healthcare and diagnostic services across a wide range of procedures and disease areas" (p. 61). ${ }^{1}$ The report specifically included the quality of primary care services within its analysis. This conclusion echoes earlier summaries of patient satisfaction studies. ${ }^{2,3}$ A number of more recent studies also identify racial and ethnic differences in patients' satisfaction with the quality of care. ${ }^{4-7}$

As Unequal Treatment suggests, disparities in the experience of the clinical encounter can be influenced by the attitudes, expectations, and behavior of both patients and physicians. Racial discordance (i.e., the situation in which the doctor and patient are from different racial or ethnic groups) can create a "social distance" between doctor and patient that reduces patients' perceptions of the quality of care and impairs their level of trust in the medical care system. ${ }^{8}$

While patient distrust may contribute to the lower levels of satisfaction reported among African American and other nonwhite patients, ${ }^{9-11}$ recent evidence also suggests that physicians may approach the clinical encounter with preconceived and sometimes stereotypical ideas and attitudes that may impact their care. ${ }^{12,13}$ For example, a study of 193 physicians suggested that those physicians perceived their black patients in more negative terms than their white patients, even after controlling for patients' income and education levels. ${ }^{14}$

If the goal of eliminating racial and ethnic disparities in health care quality is to be attained, a crucial first step is to have quality assessment instruments that will identify such differences when they exist. The concept of "quality" in medical care has at least three aspects: structure, process, and outcome. ${ }^{15,16}$ Quality instruments that measure patients' perceptions of the care process have become widely used. ${ }^{17}$ Commonly used scales that measure patients' perceptions of quality include the Consumer Assessment of Health Plans Study (CAHPS), ${ }^{18}$ administered as part of the Health Plan Employer Data and Information Set (HEDIS), ${ }^{19}$ and the 9-Item Visit Satisfaction Questionnaire (VSQ-9), developed for the Medical Outcomes Study (MOS). ${ }^{20,21}$

While CAHPS is often used to measure the quality of care received from a health plan, ${ }^{22}$ the VSQ-9 provides a measurement specifically of a patient's perception of the quality of a single office visit with a physician or other provider. $^{23,24}$ The reliability and relative ease of administration of the VSQ-9 has led to it being adopted by a number of medical groups and health services researchers to measure patient satisfaction with care. ${ }^{25,26}$ The American Medical Group Association (AMGA), a national association of large medical groups that, in aggregate, provide care to more than 50 million patients, has adapted the VSQ-9 as its recommended patient satisfaction instrument. ${ }^{27}$ 
As the VSQ-9 patient satisfaction scale is now widely used to measure this aspect of health care quality, it is essential to determine whether that scale, when used to calculate a composite score, is sufficiently sensitive in measuring racial and ethnic differences. An analysis of data from the original MOS identified racial and ethnic differences in overall patient satisfaction using a composite score from the VSQ-9 that combined different aspects of the clinical encounter. ${ }^{28}$ However, our previous report of a study of 291 patients from a single, large (>150-physician) medical group in California, also using the composite score, did not identify racial/ethnic differences in satisfaction. ${ }^{29}$ It is the purpose of this study to test whether that absence of an identified difference in satisfaction between white and nonwhite patients might be due to the manner in which the VSQ-9 score was calculated. We addressed this question by gathering data on an additional 246 patients from a variety of medical group settings. Using the combined data from a total of 537 patients, we first test for differences in satisfaction levels between white and nonwhite patients using the composite scale that includes questions pertaining to access to care as well as the quality of the physician/ patient interaction. We then reanalyze the data, separating out only those questions from the VSQ-9 that pertain to the direct physician/patient interaction.

\section{METHODS}

\section{Study Design and Population}

This study is an extension of an earlier report of 291 primary care patient visits at a single, large, multispecialty medical group that participates in a variety of managed care plans. ${ }^{29}$ The study combines data from those visits with 246 additional visits at other medical groups in the same geographic area. In addition to the large group originally studied, we approached 10 small primary care groups, ranging in size from 2 to 8 physicians. Seven of the 10 groups agreed to participate in the study; all physicians in the participating groups agreed to have their patients included in the study. All were private practice groups with no direct academic affiliation. As obstetric/ gynecology visits were included in the original sample, one of the new groups included was a strictly ObGyn group with 5 physicians. The other 7 groups were either general internal medicine or family practice. Of the approximately 65 physicians included in this study, 4 were Asian American and the remainder were white. There were no AfricanAmerican physicians either on the primary care staff of the large medical group or in primary care practice in the office clusters from which we selected our small practices. The physicians were approximately evenly split between men and women.

At each medical group a trained surveyor stood at the entrance to the office and, using a randomization protocol described elsewhere, ${ }^{30}$ approached an entering patient. After obtaining consent for participation (patient partici-
Table 1. Questions Included in the Original VSQ-9 Survey

In terms of your satisfaction, how would you rate each of the following?

$($ Excellent $=5$, Very Good $=4$, Good $=3$, Fair $=2$, Poor $=1)$

1) How long you had to wait to get an appointment

2) Convenience of the location of the office

3) Getting through to the office by phone

4) Length of time waiting at the office

5) The time spent with the doctor you saw

6) Explanation of what was done for you

7) The technical skills of the doctor you saw

8) The personal manner of the doctor you saw

9) The visit overall

pation rate was $82 \%$ ), the surveyor accompanied the patient through all aspects of the visit except entering the examination area, asking survey questions as described below. If the patient to be seen was under 18 years of age, parental consent was obtained and survey questions were addressed to the parent.

\section{Study Variables}

The dependent variable for this study is patient satisfaction with the subject office visit as measured by the VSQ-9 scale. The 9 questions included in the scale are shown in Table 1. Questions 1 to 4 pertain to satisfaction with access to care; 5 to 8 pertain to satisfaction with the direct encounter with the physician; 9 pertains to an overall assessment of satisfaction. All responses are measured on a 5-point scale, ranging from poor $=1$ to excellent $=5$. In using the scale, one can either report a mean per-item score, or the distribution of scores among the 5 categories of response. ${ }^{31}$ For this study, we report the mean, per-item response.

The surveyor asked questions 1 to 3 while the patient was in the waiting room waiting to be seen. We asked question 4, rating satisfaction with the time spent waiting at the office, as two separate questions: satisfaction with time spent waiting in the waiting room before being called in to see the physicians, and satisfaction with time spent in the examination room before the physician actually entered. We took the mean of these responses to compute a single score for this question. We asked questions 5 to 8 as soon as the patient returned to the waiting area from the examination area, and question 9 as the patient exited the facility. We have reported elsewhere the results of our test as to whether the presence of the interviewer influenced patients' responses, creating a "Hawthorne effect." 30 To do this we identified matched, case-control patients seeing the same physician for the first 100 patients from the initial sample of 291 patients, and administered a follow-up phone survey to both groups. There was no difference in satisfaction between the patients who interacted with a surveyor at the time of the original visit and those who did not. 
Because organizations theory suggests that the quality of the interaction between patient and nonphysician staff may affect the patient's perception of the quality of the direct physician/patient encounter, ${ }^{32}$ we included these measures as independent variables. As the patient interacted with individual staff members, the surveyor in our study asked the patient to rate the quality of that specific interaction. We grouped staff members encountered into nursing staff and nonnursing staff, and calculated a mean response for each group.

The principal hypothesis of this study is that the association between patients' race or ethnicity and their satisfaction ratings using the combined, 9-item scale will be different than their ratings of their direct interaction with the physician. Accordingly, we calculated a separate score for satisfaction with the direct physician encounter by taking the mean of the responses to questions 5 to 8 . Using confirmatory factor analysis, we were able to show that this 4 -item indicator is highly reliable (Cronbach's $\alpha=.88$ ). Further analysis suggested that questions 1 to 4 cannot reliably be combined into a indicator of access/convenience of care (Cronbach's $\alpha=.56$ ).

The independent variables included in the study are:

1. Patient's gender;

2. Patient's age at the time of the visit;

3. Patient's self-reported ethnicity, as a single category among those listed;

4. Patient's highest level of education completed;

5. Patients' family income;

6. Patients' estimate of the number of visits made to a physician within the past 12 months (in our earlier study, this single question was highly correlated with the SF-12 health status scale);

7. Continuity of care, measured as the percent of visits made in the previous 12 months that were to the patient's primary care physician; and

8. As described above, patients' rating of the quality of their interaction with the nursing staff and the nonnursing staff.

In measuring continuity of care, we also asked patients both about the number of visits and the length of time they had been under the care of the physician they were seeing that day. Neither variable had a significant association with reported satisfaction, and so neither is included in the model we report here.

While we noted the ethnicity and gender of the physicians included in our study, we did not attempt to control for these factors in our analysis. As the physicians in the sample were $92 \%$ white, the patient's race/ethnicity appears to be a close approximation of racial/ethnic concordance between physician and patient. Also, as the physicians were approximately equally split between men and women, and as the literature suggests that gender concordance is not a major factor in patients' perceptions of the quality of the interaction with a primary care physician, ${ }^{14}$ we did not include this variable in our analysis.

\section{Data Analysis}

We use linear regression analysis (SPSS-10; SPSS Incorporated, Chicago, Ill) to test for associations between the independent variables and the 2 dependent variables: mean 9-item satisfaction, and mean physician-specific satisfaction. In both models, we include only those independent variables found to have a significant association with the full, 9-item scale. In a separate analysis, we tested for an association between excluded variables and the physician-specific scale, and found none.

We created dummy variables from the categorical variables as follows.

- We divided family income into 3 categories of $<\$ 40,000$, $\$ 40,000$ to $\$ 80,000$, and $>\$ 80,000$, representing approximations of national income tertiles. We used the middle category as the excluded, reference category.

- We divided education completed into 3 categories of high school or less, any college, and any postgraduate work. We used any college as the reference category.

- The racial/ethnic distribution described in Table 2 indicates that there were relatively few patients in each of the nonwhite categories. Recognizing that these groups may be quite different culturally, we tested for differences in satisfaction among the various nonwhite groups using dummy variables for major nonwhite groups and black as the omitted reference group, and found none. Based on the small sample size within each group, we divided ethnicity into 2 categories: white and nonwhite, using white as the reference category.

- In our initial study, patients seen at a small (8-physician) satellite office that was part of the main medical group but geographically separate from the main facility $(>150$ physicians) reported a higher satisfaction rating on the 9-item scale after controlling for all the variables in the model. Accordingly, we created a dummy variable to indicate that the patient was seen at either the large office, or at one of the smaller offices included in the study. The large office was the reference group. A cross-tabs analysis demonstrated no clustering of patients by race/ethnicity between the single large office and the multiple small offices $(P=.29)$.

\section{RESULTS}

\section{Patient Characteristics}

A total of 537 patients completed at least part of the survey. As shown in Table 2, the patients included in this study represent an unusual study population, in that they have both high levels of education and high family income. Two thirds of the patients had graduated from college, with more than half of those having gone on to do graduate work. Consistent with this educational level, nearly three quarters of the patients reported annual family income of $\$ 60,000$ or more. These findings are not surprising, however, as the study was done in a geographic region of northern 


\section{Table 2. Characteristics of Study Subjects}

Number of subjects surveyed: 537

Number of subjects excluded due to missing variables: 57

Number of subjects included in analyses: 480

Gender, \%

Male

Female

68

Highest level of education completed, \%

Less than 8th grade

Some high school

High school graduate

Some college

College graduate

Postgraduate work

Annual family income from all sources, \%

$<\$ 10,000$

$\$ 10,000$ to $\$ 19,999$

$\$ 20,000$ to $\$ 29,999$

$\$ 30,000$ to $\$ 39,999$

$\$ 40,000$ to $\$ 49,999$

$\$ 50,000$ to $\$ 59,999$

$\$ 60,000$ to $\$ 69,999$

$\$ 70,000$ to $\$ 79,999$

$\$ 80,000$ or more

Race/ethnicity, \%

White

Black

Mexican

Hispanic, other than Mexican

Asian/Pacific Islander

Other

Size of medical group in which visit took place, \%

$>150$ physicians (1 medical group)

5 to 10 physicians (3 medical groups)

$<5$ physicians (5 medical groups)

Mean (SD) of continuous variables

Age of patient, y

Number of physician visits per year

Percent of visits with primary care physician

Satisfaction with nursing staff

Satisfaction with nonnursing staff

9-item satisfaction scale (per item)

4-item physician-specific satisfaction scale (per item)

$\mathrm{SD}$, standard deviation.

California that has one of the highest educational and income levels in the country. In addition, the study population is skewed from the general population in the area in that all the medical groups included in the study require either proof of insurance or advance payment before accepting new patients.

Of the 537 patients in the study, one or more variables were missing from 57 cases, or $11 \%$. Of these, 10 cases were missing data required to compute the satisfaction scales used as our dependent variable. For the additional 47 cases, the most frequent missing variable was the subject's reported satisfaction with the nonphysician staff $(n=31)$. To test for the effect of missing data, we replaced missing variables with mean values (continuous) or median values (discrete) of all missing variables, and reanalyzed the data. The overall results of the analysis were not changed by using these imputed data for the missing cases.

\section{Data Analysis}

Table 3 shows the results of the two separate regressions conducted in this analysis. The results include both the unstandardized (b) as well as the standardized ( $\beta$ ) regression coefficients. The first question to ask is whether nonwhite race/ethnicity shows an association with the full, 9-item satisfaction score after controlling for the other independent variables in the model. As shown in column 1 , with an adjusted r-square of .36, the variables included account for $36 \%$ of the variance in mean satisfaction. The standardized regression coefficients ( $\beta$ ), the absolute value of which provides a comparison of the relative magnitude of the effects of the different variables, indicates that age, overall level of health, seeing a physician in one of the smaller offices, and quality of the interaction with the nonphysician staff have the strongest associations with patient satisfaction. The patient's level of education had a smaller but significant effect. Patient gender did not have a significant effect, so is not included in the model. A separate analysis tested for different outcomes for children (age $<18$, for whom the parent was surveyed) and adults, and found none.

The analysis using this model did not identify a significant difference between white and nonwhite patients in their overall satisfaction with their visit. This finding duplicates that reported in our initial study. ${ }^{30}$ If the 9-item satisfaction scale is a valid measure of racial/ethnic differences in satisfaction, this finding would suggest that white and nonwhite patients in the community studied report the same level of satisfaction with care.

Column 2 of Table 3 shows the association between the above variables and the 4-item, physician-specific scale. As with the earlier model, the variables included account for a substantial amount of the variance $(25 \%)$ in this satisfaction scale. Age, education, and health status continue to have significant, though somewhat weaker, effects. The size of the facility in which the visit took place no longer shows an effect, suggesting that patients' perceptions of physician quality is independent of the organizational size of the practice setting after controlling for other aspects of the visit. The patients' satisfaction with the nonphysician staff continues to show a strong association with their perception of the quality of the direct physician encounter, even though most (but not all) of the staff interactions measured by these variables took place before the patient was seen by the physician.

In this second model, measuring only satisfaction with the direct physician encounter and controlling for demographic variables and aspects of the visit not involving the physician, nonwhite patients rate the quality of their interaction with the physician significantly lower than white patients seeing the same groups of physicians. The 
Table 3. Factors Associated with Mean Patient Satisfaction Using the 9-Item Scale and the 4-Item Physician-specific Scale

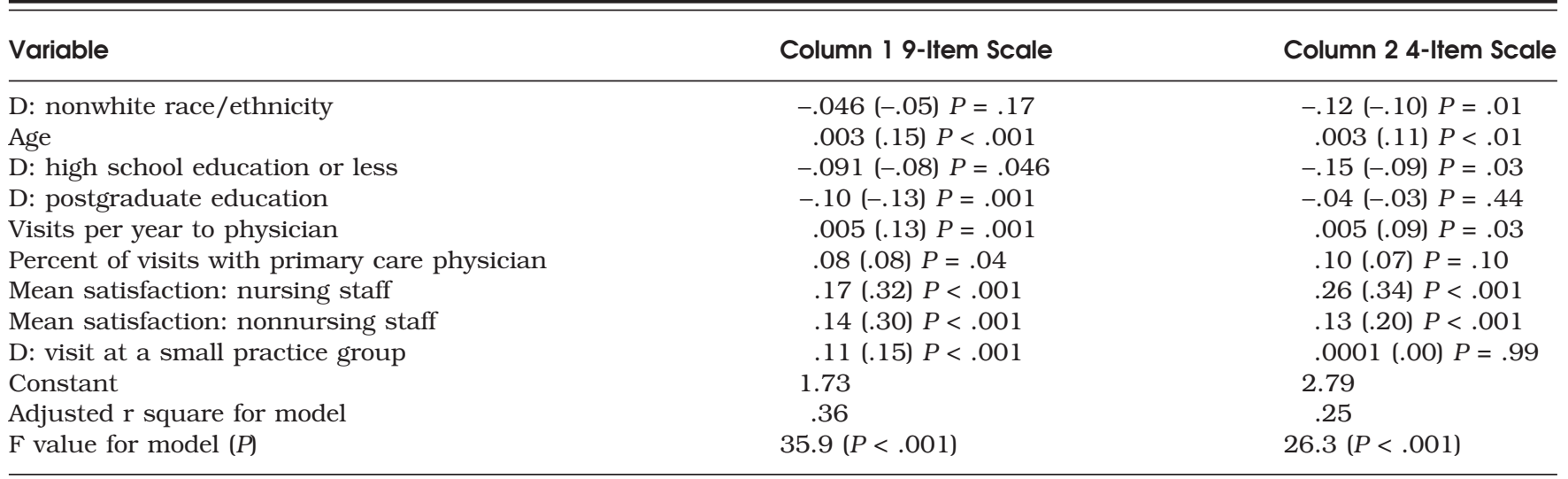

Results of ordinary least squares regression analysis $\mathrm{N}=480$. Shows $b(\beta), \mathrm{P}=(D=$ dummy variable $)$.

standardized regression coefficients $(\beta)$ indicate that the relative magnitude of this association is stronger than that for education, health status, and continuity of previous care, and comparable to the effect of age.

\section{DISCUSSION}

A recent report recommends that the quality of physician office care be measured nationally using a standardized, composite score that combines a series of indicators. ${ }^{33}$ The results of this study suggest that using such a composite score may not be sufficiently sensitive to identify racial or ethnic differences in patient satisfaction in certain settings. Among the patients in this study, representing a generally affluent, well-educated population with full health insurance coverage, nonwhites reported a lower level of satisfaction with their direct physician/patient interaction than white patients. However, these differences were not apparent when using the composite 9-item scale.

This study confirms that the interpersonal dynamics that occur between physician and patient play a role in creating the racial and ethnic differences in satisfaction that have been observed. From a quality improvement perspective, it is crucial to have measurement instruments that are sufficiently sensitive to racial and ethnic differences among patient populations if the national policy goal of eliminating racial and ethnic differences is to be achieved. For purposes of improving the quality of the physicianpatient interaction, analysis should be done using those items within the scale that measure the quality of the physician/patient interaction directly, using other measures (e.g., waiting time, staff courtesy) as control variables.

It is not possible, however, to look for racial or ethnic differences in care if a medical group or organization does not include the patient's racial or ethnic group as part of the standard demographic patient database. As part of its National Standards for Culturally and Linguistically Appropriate Services in Health Care, the U.S. Department of Health and Human Services has adopted the following:
Standard 10. Health care organizations should ensure that data on the individual patient's/consumer's race, ethnicity, and spoken and written language are collected in health records, integrated into the organization's management information systems, and periodically updated. ${ }^{34}$

Whether medical care providers should routinely gather information regarding patient race or ethnicity has been a subject of recent debate. None of the medical groups included in this study includes that information in its database. Racial and ethnic differences in patient satisfaction and other measures of health care quality do not occur only among low-income patients. As this study suggests, those differences span the socioeconomic spectrum. In this study, in which we asked patients to identify their race or ethnicity as part of a publicly administered survey, 536 out of 537 patients provided this information. Concerns that patients will object to or be uncomfortable with efforts to gather data regarding race or ethnicity do not seem to be well founded.

Once racial or ethnic differences in care have been identified, the question arises as to the source(s) of those differences. While this study identifies such differences, it does not identify how the differences originate. Certainly there are at least two possibilities.

1. Nonwhite patients approach their interaction with the physician with different attitudes and expectations, which are reflected in their lower expressed levels of satisfaction. Principal among these is the general level of mistrust of the medical care system described above.

2. Physicians may treat nonwhite patients differently than white patients in ways that adversely impact patient satisfaction. These differences may reflect the stereotypes and unconscious attitudes which physicians may bring to the racially discordant patient encounter.

If the results of this study were due principally to patients' preconceived attitudes toward the medical care system, we would expect nonwhite patients to be less satisfied with their interaction with the physician as well as 
the nonphysician staff. To test for this potentiality, we ran separate regression analyses looking for an association between the independent variables in our model and patients' mean satisfaction with the nursing staff encountered, and their mean satisfaction with the nonnursing staff encountered. In neither case did any of the demographic variables, including patient's race/ethnicity, have any statistical association with these satisfaction outcomes. There are thus no indirect effects of demographic variables through satisfaction with the nonphysician staff. Among the patients included in this study, nonwhite patients were no different than white patients in their perceptions of the quality of their interactions with the nonphysician staff. These results suggest that the observed racial/ethnic differences in patient satisfaction with physician-provided care reflect differences in the way the physicians studied approach patients from different racial or ethnic groups. Whether this difference reflects bias on the part of the physician, or rather simply physicians' unfamiliarity with the differing dynamics of physician-patient communication in the racially or ethnically discordant encounter, cannot be answered by this study, and deserves further research.

This study has a number of weaknesses that may affect our ability to generalize from these findings. Our study population is from a relatively small number of medical groups, from a relatively small geographic area. It is possible that, in a larger study population, the full 9-item satisfaction scale may have identified racial/ethnic differences. In addition, the highly skewed nature of the socioeconomic background of the patients studied makes it difficult to predict what results might be found in studies of patients more representative of the general population. Similarly, the inclusion of obstetrics/gynecology among the primary care specialties studied contributed to the skewed gender distribution of our study sample, with two-thirds of the patients surveyed being female. However, it is not uncommon for individual medical groups to conduct patient satisfaction surveys on study populations of approximately the same size as those reported here. In that situation, it would seem prudent to analyze those data in ways that would identify specific racial/ethnic differences in the direct physician/patient encounter.

This research was supported in part by grant 1 RO3 HSO9350 from the U.S. Agency for Healthcare Research and Quality.

\section{REFERENCES}

1. Institute of Medicine. Unequal Treatment: Confronting Racial and Ethnic Disparities in Health Care. Washington, DC: National Academy of Sciences; 2002. Available at: http://www.nap.edu/ catalog/10260.html. Accessed July 24, 2003.

2. Hall JA, Dornan MC. Patient sociodemographic characteristics as predictors of satisfaction with medical care: a meta-analysis. Soc Sci Med. 1990;30:811-8.

3. Sitzia J, Wood N. Patient satisfaction: a review of issues and concepts. Soc Sci Med. 1997;45:1829-43.
4. Carlson MJ, Blustein J, Fiorentino N, Prestianni F. Socioeconomic status and dissatisfaction among HMO enrollees. Med Care. 2000; 38:508-16.

5. Zaslavsky AM, Hochheimer JN, Schneider EC, et al. Impact of sociodemographic case mix on the HEDIS measures of health plan quality. Med Care. 2000;38:981-92.

6. Shi L. Experience of primary care by racial and ethnic groups in the United States. Med Care. 1999;37:1068-77.

7. Morales LS, Elliott MN, Weech-Maldonado R, Spritzer KL, Hays RD. Differences in CAHPS adult survey reports and ratings by race and ethnicity: an analysis of the National CAHPS benchmarking data 1.0. Health Serv Res. 2001;36:595-617.

8. Malat J. Social distance and patients' rating of healthcare providers. $\mathrm{J}$ Health Soc Behav. 2001;42:360-72.

9. LaVeist TA, Nickerson KJ, Bowie JV. Attitudes about racism, medical mistrust, and satisfaction with care among African American and white cardiac patients. Med Care Res Rev. 2000;57(suppl 1): 146-61.

10. Blendon RJ, Scheck AC, Donelan K, et al. How white and African Americans view their health and social problems. Different experiences, different expectations. JAMA. 1995;273:341-6.

11. Doescher MP, Saver BG, Franks P, Fiscella K. Racial and ethnic disparities in perceptions of physician style and trust. Arch Fam Med. 2000;9:1156-63

12. van Ryn M. Research on the provider contribution to race/ethnicity disparities in medical care. Med Care. 2002;40(1 suppl):140-51.

13. Cooper-Patrick L, Gallo JJ, Gonzales JJ, et al. Race, gender, and partnership in the patient-physician relationship. JAMA. 1999;282: 583-9.

14. van Ryn M, Burke J. The effect of patient race and socio-economic status on physicians' perceptions of patients. Soc Sci Med. 2000; 50:813-28.

15. Donabedian A. Evaluating the quality of medical care. Milbank Mem Fund Q. 1966;44(suppl):166-206.

16. Donabedian A. The Definition of Quality and Approaches to Its Assessment. Ann Arbor, Mich: Health Administration Press; 1980.

17. Cleary PD, Edgman-Levitan S. Health care quality. Incorporating consumer perspectives. JAMA. 1997;278:1608-12.

18. Agency for Health Care Research and Quality. U.S. Department of Health and Human Services. Consumer Assessment of Health Plans (CAHPS). Available at: http://www.ahrq.gov/qual/cahpfact.htm. Accessed July 24, 2003.

19. National Committee for Quality Assurance. The Health Plan Employer Data and Information Set. Available at: http://www.ncqa.org/ Programs/HEDIS/index.htm. Accessed July 24, 2003.

20. Tarlov AR, Ware JE Jr, Greenfield S, Nelson EC, Perrin E, Zubkoff M. The Medical Outcomes Study. An application of methods for monitoring the results of medical care. JAMA. 1989;262:925-30.

21. Rubin HR, Gandek B, Rogers WH, Kosinski M, McHorney CA, Ware JE. Patients' ratings of outpatient visits in different practice settings. Results from the Medical Outcomes Study. JAMA. 1993; 270:835-40.

22. Weisman CS, Henderson JT, Schifrin E, Romans M, Clancy CM. Gender and patient satisfaction in managed care plans: analysis of the 1999 HEDIS/CAHPS 2.0H Adult Survey. Womens Health Issues. 2001;11:401-15.

23. Ware JE, Hays RD. Methods for measuring patient satisfaction with specific medical encounters. Med Care. 1988;26:393.

24. Ware JE, Snyder MK, Wright WR, Davies AR. Defining and measuring patient satisfaction with medical care. Eval Program Plann. 1988;6:247-63.

25. Murray-Garcia JL, Selby JV, Schmittdiel J, Grumbach K, Quesenberry CP Jr. Racial and ethnic differences in a patient survey: patients' values, ratings, and reports regarding physician primary care performance in a large health maintenance organization. Med Care. 2000;38:300-10.

26. Jackson JL, Chamberlin J, Kroenke K. Predictors of patient satisfaction. Soc Sci Med. 2001;52:609-20. 
27. American Medical Group Association. Patient Satisfaction Survey. Available at: http://www.amga.org/QMR/PSAT/survey_psat.asp. Accessed July 24, 2003.

28. Meredith LS, Siu AL. Variation and quality of self-report health data. Asians and Pacific Islanders compared with other ethnic groups. Med Care. 1995;33:1120-31.

29. Barr DA, Vergun P, Barley SR. Problems in using patient satisfaction data to assess the quality of care provided by primary care physicians. J Clin Outcomes Manag. 2000;7:19-24.

30. Barr DA, Vergun P. Using a new method of gathering patient satisfaction data to assess the effects of organizational factors on primary care quality. Jt Comm J Qual Improv. 2000;26:71323.
31. Davies AR, Ware JE. GHAA's Consumer Satisfaction Survey and User's Manual. Washington, DC: Group Health Association of America; 1991.

32. Barr DA. The effects of organizational structure on primary care outcomes under managed care. Ann Intern Med. 1995;122:353-9.

33. Zaslavsky AM, Shaul JA, Zaborski LB, Cioffi MJ, Cleary PD. Combining Health Plan Performance Indicators into Simpler Composite Measures. Health Care Financ Rev. 2002;23:101-15.

34. Office of Minority Health, U.S. Department of Health and Human Services. National Standards for Culturally and Linguistically Appropriate Services in Health Care. Executive Summary. March 2001. Available at: http://www.omhrc.gov/clas/. Accessed July 24, 2003. 